\title{
Effect of Urban Compost, Sewage Sludge and Poultry Manure with Chemical Fertilizers on Yield, Nutrient Content and Uptake in Brinjal- Cauliflower Cropping System
}

\author{
R. Saikumar* and K. Jeevan Rao
}

Department of Soil Science, College of Agriculture, PJTSAU, Hyderabad-500030, India *Corresponding author

\section{A B S T R A C T}

\section{Keywords}

Brinjal,

Cauliflower,

Organic manures,

Nutrient uptake, Yield.

Article Info

Accepted:

19 May 2017

Available Online:

10 June 2017
An experiment was conducted during kharif 2013 and rabi 2013-14 to evaluate the best combination of organic and inorganic sources of nutrients for maximizing yield. Combined application of poultry manure $5 \mathrm{t} \mathrm{ha}^{-1}$ and $75 \%$ recommended dose of NPK recorded higher fruit yield $\left(33.6 \mathrm{t} \mathrm{ha}^{-1}\right)$ and curd yield $\left(29.3 \mathrm{t} \mathrm{ha}^{-1}\right)$ followed by combined application of sewage sludge $5 \mathrm{t} \mathrm{ha}^{-1}$ and $75 \%$ recommended dose of NPK. Experimental results revealed that the concentration and uptake of major nutrients by brinjal and cauliflower plant parts were significantly influenced by the application of organic manures along with inorganic fertilizers. In both the seasons highest values for net income noted for treatment involving combined use of poultry manure $5 \mathrm{t} \mathrm{ha}^{-1}+75 \% \mathrm{RDF}\left(\mathrm{Rs} .301369 \mathrm{ha}^{-1}\right)$, whereas the highest benefit: cost ratio (2.27) were noted for sewage sludge $5 \mathrm{t} \mathrm{ha}^{-1}+$ $75 \%$ RDF.

\section{Introduction}

The imbalanced and skewed application of NPK accompanied by restricted use of organic manures and micronutrients have made soils not only deficient in the nutrients, but also deteriorated the soil health, resulting in declining of the crop response to the application of fertilizers. On the other hand, Organic manures alone may not be able to meet the nutrient requirement of high yielding crops to produce the required food grains for the burgeoning human population due to their low nutrient contents and slow rates of nutrient release. Under this circumstance, integration of chemical and organic sources and their management have shown promising results not only in sustaining the productivity but have also proved to be effective in maintaining soil health and enhancing nutrient use efficiency (Laxminarayana et al., 2011; Kumar Mukesh et al., 2012). The supplementary and complementary use of organic manures and inorganic fertilizers augment the efficiency of both substances to sustain soil productivity. Keeping in view the significance of organic manures in maintaining the soil health and importance of brinjal and cauliflower in human nutrition an attempt was made to critically examine the 
use of poultry manure, sewage sludge and urban compost along with chemical fertilizers to obtain better yields and to maintain better soil health in brinjal-cauliflower cropping system under integrated nutrient management.

\section{Materials and Methods}

A field experiment was conducted at College farm, Rajendranagar, during kharif 2013 and rabi 2013-14. The soil was sandy loam in texture and slightly alkaline in reaction. It was low in available $\mathrm{N}$, medium in available $\mathrm{P}, \mathrm{K}$ and low in organic carbon (Table 1). The experiment was laid out in a randomized block design with 14 treatment combinations, each being replicated thrice consisting of two levels of each of sewage sludge,urban compost and poultry manure $2.5,5.0 \mathrm{t} \mathrm{ha}^{-1}$ and combination of 75 percent RDF. The organic manures i.e., poultry manure (PM), urban compost (UC) and sewage sludge (SS) procured from poultry station, Rajendranagar, Hyderabad, SELICO private company gandemguda, Rangareddy and Amberpet sewage treatment plant, respectively. All theses manures were analyzed for their chemical composition viz., $\mathrm{N}, \mathrm{P}, \mathrm{K}, \mathrm{OC}, \mathrm{pH}$, EC and available micronutrients (Table 2). All these manure were applied as per the treatments. N, P and $\mathrm{K}$ were applied through Urea, SSP and MOP, respectively while the total quantity of phosphorus and potassium were applied as basal and nitrogen was applied in three equal splits viz. $1 / 3$ as basal, $1 / 3$ at flowering and the remaining $1 / 3$ at fruit formation to brinjal. Thirty days old seedlings of brinjal (hybrid) were transplanted on ridges at a spacing of $60 \mathrm{~cm} \times 60 \mathrm{~cm}$. During rabi season cauliflower was grown to know the manures and fertilizers effect on soil to assay the cumulative and residual effects on cauliflower from earlier brinjal crop. Each earlier treatments plot was divided into two; among them, one plot was used to get cumulative effect on cauliflower, other one was used to get residual effect on cauliflower. However, the crop was grown without addition of any manure and fertilizer for residual sub plot. Cumulative sub plot was applied with fertilizers and manures as per the crop recommendation and treatments. In both seasons Plants samples were collected at harvest stage; yields of fruit/ head and plant were recorded separately after thoroughly sun drying. These samples were air dried and later oven dried at $60^{\circ} \mathrm{C}$ and ground to fine power using stainless steel willy mill and analyzed for $\mathrm{N}, \mathrm{P}$ and $\mathrm{K}$ content by adopting standard methods as micro kjeldahl method, Vanado molybdate phosphoric method and flame photometry, respectively. After determination of nutrient concentration in edible part and plant separately, these values were multiplied by corresponding dry matter yield to obtain nutrient uptake (Table 3). Data was analyzed statistically to test significances and the treatments are tested at five percent level of significance. The analysis was carried out by the methodology as described by Panse and Sukhatme (1976).

\section{Results and Discussion}

\section{Brinjal fruit yield (kharif, 2013)}

Brinjal performance was significantly increased by the application of sewage sludge, urban compost, poultry manure along with 75 percent recommended dose of fertilizers as compared to control. Combined application of organic manures and inorganic fertilizers proved better in increasing brinjal fruit size and yield. Significantly higher fruit yield were recorded for treatment supplying poultry manure $5.0 \mathrm{t} \mathrm{ha}^{-1}$ in combination with 75 percent recommended dose of NPK (33.6 t ha $\left.{ }^{-1}\right)$ followed by treatment supplying sewage sludge $5.0 \mathrm{t} \mathrm{ha}^{-1}$ in combination with 75 percent recommended dose of NPK (32.5 $\mathrm{t} \mathrm{ha}^{-1}$ ) (Table 4). Higher fruit yield was observed with combined application of inorganic and 
organic sources of nutrients may be attributed to adequate and continuous supply of essential elements at initial stage by inorganic fertilizers and organic manures at later stage, improved soil properties, development of proliferous root system, increased metabolic activity, greater synthesis, translocation and accumulation of carbohydrates. Similar results were also reported by Narayan et al., (2004); Rakhonde et al., (2015) and Singh Rana Veer et al., (2015).

Cauliflower fresh head yield (rabi, 201314)

In the second season of cumulative and residual effect on rabi 2014 cauliflower, significantly increase yield of $29.3 \mathrm{t} \mathrm{ha}^{-1}$ and $22.0 \mathrm{t} \mathrm{ha}^{-1}$ was recorded with poultry manure
$5.0 \mathrm{t} \mathrm{ha}^{-1}+75 \% \mathrm{RDF}$ ), followed by SS $5.0 \mathrm{t}$ $\mathrm{ha}^{-1}+75 \%$ RDF $28.7 \mathrm{t} \mathrm{ha}^{-1}$ and $21.8 \mathrm{t} \mathrm{ha}^{-1}$ (Table 4). Integrated manures and fertilizer applied plots showed good response to yield and individually manure treated plots showed significantly lesser yield than combined applications. The relative increase in yield by combined application of poultry manure and fertilizers of could be attributed to improvement in soil structure, nutrient retention and water for plant use.

\section{Nutrient concentrations and uptake}

In both the seasons, the highest $\mathrm{N}$ concentration and uptake were recorded with the poultry manure $5.0 \mathrm{t}$ ha- 1 along with $75 \%$ recommended dose of fertilizers.

Table.1 Salient Characteristics of experimental Soil

\begin{tabular}{|c|c|c|}
\hline S.No & Characteristics of soil & Value \\
\hline I & Physical properties & \\
\hline a. & Bulk density $\left(\mathrm{Mg} \mathrm{m}^{-3}\right)$ & 1.40 \\
\hline \multirow[t]{5}{*}{ b. } & Mechanical composition (\%) & \\
\hline & Sand & 74.80 \\
\hline & Silt & 5.85 \\
\hline & Clay & 19.35 \\
\hline & Textural class & Sandy loam \\
\hline c. & Water holding capacity $(\%)$ & 21.40 \\
\hline II. & Physico-chemical properties & \\
\hline a. & Soil reaction $(\mathrm{pH})$ & 7.50 \\
\hline b. & Electrical conductivity $(\mathrm{EC})\left(\mathrm{dSm}^{-1}\right)$ & 0.35 \\
\hline c. & Cation exchange capacity (CEC) $\left[\mathrm{cmol}\left(\mathrm{p}^{+}\right) \mathrm{kg}^{-1}\right]$ & 19.20 \\
\hline III. & Chemical properties & \\
\hline a. & Organic carbon $(\%)$ & 0.44 \\
\hline b. & Available nitrogen $\left(\mathrm{kg} \mathrm{ha}^{-1}\right)$ & 203.8 \\
\hline c. & Available phosphorus $\left(\mathrm{kg} \mathrm{ha}^{-1}\right)$ & 24.0 \\
\hline $\mathrm{d}$ & Available potassium $\left(\mathrm{kg} \mathrm{ha}^{-1}\right)$ & 234 \\
\hline III & Enzymatic activity & \\
\hline a. & Urease $\left(\mu \mathrm{g}\right.$ of $\mathrm{NH}_{4}^{+}-\mathrm{N}$ released $\mathrm{g}^{-1}$ soil $\left.\mathrm{h}^{-1}\right)$ & 3.30 \\
\hline b. & Dehydrogenase $\left(\mu \mathrm{g}\right.$ of TPF produced $\mathrm{g}^{-1}$ soil d $\left.^{-1}\right)$ & 1.80 \\
\hline c. & Acid phosphatase ( $\mu \mathrm{g}$ of $\mathrm{p}$-nitrophenol released $\mathrm{g}^{-1}$ soil $^{-1}$ ) & 60.0 \\
\hline $\mathrm{d}$ & Alkaline phosphatase ( $\mu \mathrm{g}$ of $\mathrm{p}$-nitrophenol released $\mathrm{g}^{-1}$ soil $\left.^{-1}\right)$ & 63.0 \\
\hline
\end{tabular}


Table.2 Characteristics of urban compost, sewage sludge and poultry manure

\begin{tabular}{|c|c|c|c|c|}
\hline S.No & Character & $\begin{array}{c}\text { Urban } \\
\text { compost }\end{array}$ & Sewage sludge & $\begin{array}{l}\text { Poultry } \\
\text { manure }\end{array}$ \\
\hline \multicolumn{5}{|c|}{ Physico- chemical properties } \\
\hline 1 & $\mathrm{pH}$ & 7.13 & 6.80 & 7.10 \\
\hline 2 & $\mathrm{EC}\left(\mathrm{dS} \mathrm{m} \mathrm{m}^{-1}\right)$ & 1.52 & 1.60 & 1.20 \\
\hline 3 & $\mathrm{OC}(\%)$ & 23.00 & 29.00 & 33.10 \\
\hline \multicolumn{5}{|c|}{ Total major nutrient status (\%) } \\
\hline 4 & $\mathrm{~N}$ & 0.90 & 1.60 & 2.80 \\
\hline 5 & $\mathrm{P}$ & 0.32 & 0.93 & 1.38 \\
\hline 6 & $\mathrm{~K}$ & 0.54 & 0.60 & 1.46 \\
\hline \multicolumn{5}{|c|}{ DTPA extractable micronutrients and heavy metals $\left(\mathrm{mg} \mathrm{kg}^{-1}\right)$} \\
\hline 7 & $\mathrm{Fe}$ & 189.0 & 260.0 & 324.0 \\
\hline 8 & $\mathrm{Mn}$ & 30.0 & 55.0 & 76.5 \\
\hline 9 & $\mathrm{Zn}$ & 19.0 & 28.8 & 59.0 \\
\hline 10 & $\mathrm{Cu}$ & 14.0 & 19.4 & 16.2 \\
\hline 11 & $\mathrm{Cd}$ & 1.30 & 1.56 & 0.56 \\
\hline 12 & $\mathrm{Ni}$ & 1.42 & 5.30 & 2.69 \\
\hline 13 & $\mathrm{Cr}$ & 5.29 & 6.24 & 2.13 \\
\hline 14 & $\mathrm{~Pb}$ & 4.23 & 5.60 & 2.04 \\
\hline \multicolumn{5}{|c|}{ Total micronutrients and heavy metals $\left(\mathrm{mg} \mathrm{kg}^{-1}\right)$} \\
\hline 15 & $\mathrm{Fe}$ & 3250.0 & 6290.0 & 1270.0 \\
\hline 16 & $\mathrm{Mn}$ & 210.0 & 758.0 & 298.0 \\
\hline 17 & $\mathrm{Zn}$ & 81.0 & 423.4 & 80.0 \\
\hline 18 & $\mathrm{Cu}$ & 87.9 & 345.6 & 64.2 \\
\hline 19 & $\mathrm{Cd}$ & 19.5 & 60.8 & 18.0 \\
\hline 20 & $\mathrm{Ni}$ & 12.2 & 61.5 & 15.0 \\
\hline 21 & $\mathrm{Cr}$ & 58.2 & 99.4 & 10.2 \\
\hline 22 & $\mathrm{~Pb}$ & 54.0 & 120.0 & 73.2 \\
\hline
\end{tabular}


Table.3 Effect of urban compost, sewage sludge and poultry manure with fertilizers on dry matter $\left(\mathrm{kg} \mathrm{ha}^{-1}\right)$ yield in brinjalCauliflower cropping system

\begin{tabular}{|c|c|c|c|c|c|c|}
\hline \multirow[b]{2}{*}{ Treatments } & \multicolumn{2}{|c|}{ Brinjal } & \multicolumn{2}{|c|}{ Cumulative cauliflower } & \multicolumn{2}{|c|}{ residual cauliflower } \\
\hline & $\begin{array}{l}\text { plant dry } \\
\text { matter }\end{array}$ & $\begin{array}{l}\text { fruit dry } \\
\text { matter }\end{array}$ & $\begin{array}{l}\text { plant dry } \\
\text { matter }\end{array}$ & head dry matter & $\begin{array}{l}\text { plant dry } \\
\text { matter }\end{array}$ & head dry matter \\
\hline T1-Control & 1450 & 1280 & 920 & 851 & 890 & 770 \\
\hline T2-RDF & 1690 & 1550 & 1256 & 2100 & 780 & 1090 \\
\hline T3-UC@ $2.5 \mathrm{t} \mathrm{ha}^{-1}$ & 1310 & 1120 & 1060 & 1800 & 860 & 1400 \\
\hline T4-UC@5 tha ${ }^{-1}$ & 1550 & 1390 & 1350 & 1950 & 1050 & 1625 \\
\hline T5-SS@ $2.5 \mathrm{tha}^{-1}$ & 1598 & 1440 & 1410 & 2060 & 1080 & 1660 \\
\hline T6-SS@5 tha ${ }^{-1}$ & 1918 & 1742 & 1530 & 2255 & 1210 & 1880 \\
\hline T7-PM@ $2.5 \mathrm{tha}^{-1}$ & 1620 & 1580 & 1486 & 2170 & 1160 & 1700 \\
\hline T8-PM@5 tha ${ }^{-1}$ & 2000 & 1910 & 1680 & 2460 & 1290 & 2000 \\
\hline T9- UC @ $2.5 \mathrm{t} \mathrm{ha}^{-1}+75 \% \mathrm{RDF}$ & 1990 & 1860 & 1500 & 2200 & 1020 & 1710 \\
\hline T10-UC@ $5 \mathrm{tha}^{-1}+75 \%$ RDF & 2430 & 2210 & 1656 & 2324 & 1100 & 1800 \\
\hline T11-SS@2.5 t ha ${ }^{-1}+75 \%$ RDF & 2528 & 2413 & 1830 & 2455 & 1280 & 1910 \\
\hline T12-SS@5 tha ${ }^{-1}+75 \%$ RDF & 2720 & 2640 & 1968 & 2649 & 1410 & 2130 \\
\hline T13-PM @ $2.5 \mathrm{t} \mathrm{ha}^{-1}+75 \% \mathrm{RDF}$ & 2620 & 2458 & 1750 & 2580 & 1315 & 1980 \\
\hline T14-PM @ $5 \mathrm{tha}^{-1}+75 \% \mathrm{RDF}$ & 2930 & 2790 & 2100 & 2810 & 1640 & 2280 \\
\hline Mean & 2025 & 1885 & 1536 & 2190 & 1149 & 1710 \\
\hline S.E m $( \pm)$ & 42 & 44 & 33 & 72 & 31 & 58 \\
\hline $\mathrm{CD}(0.05)$ & 122 & 128 & 97 & 208 & 89 & 168 \\
\hline
\end{tabular}

Note: RDF: recommended dose of fertilizer UC: urban compost SS: sewage sludge PM: poultry manure 
Table.4 Effect of urban compost, sewage sludge and poultry manure with fertilizers on yield in brinjal-cauliflower cropping system

\begin{tabular}{|c|c|c|c|}
\hline \multirow[b]{3}{*}{ Treatments } & \multicolumn{3}{|c|}{ fresh fruit/ head yield $\left(t h^{-1}\right)$} \\
\hline & \multirow[t]{2}{*}{ Brinjal (kharif, 2013) } & \multicolumn{2}{|c|}{ Cauliflower (rabi, 2014) } \\
\hline & & Cumulative & Residual \\
\hline $\mathrm{T}_{1}$ - Control & 16.0 & 13.0 & 9.4 \\
\hline $\mathrm{T}_{2}-\mathrm{RDF}$ & 27.0 & 26.1 & 12.0 \\
\hline $\mathrm{T}_{3}-\mathrm{UC} @ 2.5 \mathrm{tha}^{-1}$ & 21.0 & 19.1 & 15.0 \\
\hline $\mathrm{T}_{4}-\mathrm{UC} @ 5 \mathrm{tha}^{-1}$ & 24.0 & 20.0 & 16.4 \\
\hline $\mathrm{T}_{5}-\mathrm{SS} @ 2.5 \mathrm{tha}^{-1}$ & 23.0 & 22.0 & 17.0 \\
\hline $\mathrm{T}_{6^{-}} \mathrm{SS} @ 5 \mathrm{tha}^{-1}$ & 25.6 & 24.6 & 19.6 \\
\hline $\mathrm{T}_{7}-\mathrm{PM} @ 2.5 \mathrm{tha}^{-1}$ & 24.3 & 23.0 & 18.0 \\
\hline $\mathrm{T}_{8}-\mathrm{PM} @ 5 \mathrm{tha}^{-1}$ & 26.5 & 26.0 & 20.0 \\
\hline $\mathrm{T}_{9^{-}} \mathrm{UC} @ 2.5 \mathrm{tha}^{-1}+75 \% \mathrm{RDF}$ & 27.7 & 24.0 & 16.7 \\
\hline $\mathrm{T}_{10^{-}} \mathrm{UC} @ 5 \mathrm{t} \mathrm{ha}^{-1}+75 \% \mathrm{RDF}$ & 29.0 & 25.8 & 18.0 \\
\hline $\mathrm{T}_{11^{-}} \mathrm{SS} @ 2.5 \mathrm{t} \mathrm{ha}^{-1}+75 \% \mathrm{RDF}$ & 28.8 & 27.1 & 19.0 \\
\hline $\mathrm{T}_{12}-\mathrm{SS} @ 5 \mathrm{tha}^{-1}+75 \% \mathrm{RDF}$ & 32.5 & 28.7 & 21.8 \\
\hline $\mathrm{T}_{13^{-}} \mathrm{PM} @ 2.5 \mathrm{t} \mathrm{ha}^{-1}+75 \% \mathrm{RDF}$ & 30.0 & 27.4 & 19.2 \\
\hline $\mathrm{T}_{14^{-}} \mathrm{PM} @ 5 \mathrm{t} \mathrm{ha}^{-1}+75 \% \mathrm{RDF}$ & 33.6 & 29.3 & 22.0 \\
\hline Mean & 26.4 & 24.0 & 17.4 \\
\hline S.E m( $( \pm)$ & 1.12 & 0.61 & 1.10 \\
\hline $\mathrm{CD}(0.05)$ & 3.24 & 1.77 & 3.13 \\
\hline
\end{tabular}

Note: RDF: recon 
Table.5 Effect of urban compost, sewage sludge and poultry manure with fertilizers on nutrient concentration and uptake $\left(\mathrm{kg}^{-1} \mathrm{~h}^{-1}\right)$ in Brinjal-cauliflower cropping system

\begin{tabular}{|c|c|c|c|c|c|c|c|c|c|c|c|c|c|c|c|c|c|c|}
\hline \multirow{3}{*}{ Treatments } & \multicolumn{6}{|c|}{ brinjal } & \multicolumn{6}{|c|}{ cauliflower (cumulative) } & \multicolumn{6}{|c|}{ residual } \\
\hline & \multicolumn{3}{|c|}{ Nutrient (\%) } & \multicolumn{3}{|c|}{ uptake } & \multicolumn{3}{|c|}{ Nutrient (\%) } & \multicolumn{3}{|c|}{ uptake } & \multicolumn{3}{|c|}{ Nutrient (\%) } & \multicolumn{3}{|c|}{ uptake } \\
\hline & $\mathbf{N}$ & $\mathbf{P}$ & $\mathbf{K}$ & $\mathbf{N}$ & $\mathbf{P}$ & $\mathbf{K}$ & $\mathbf{N}$ & $\mathbf{P}$ & $\mathbf{K}$ & $\mathbf{N}$ & $\mathbf{P}$ & $\mathbf{K}$ & $\mathbf{N}$ & $\mathbf{P}$ & $\mathbf{K}$ & $\mathbf{N}$ & $\mathbf{P}$ & $\mathbf{K}$ \\
\hline T1-Control & 1.4 & 0.20 & 1.30 & 20.3 & 2.9 & 18.9 & 1.20 & 0.20 & 1.57 & 11.0 & 1.8 & 14.4 & 1.23 & 0.16 & 1.59 & 10.9 & 1.4 & 14.2 \\
\hline T2-RDF & 2.1 & 0.50 & 1.60 & 35.5 & 8.4 & 27.0 & 1.90 & 0.32 & 2.54 & 23.2 & 4.0 & 31.9 & 1.39 & 0.21 & 1.78 & 10.8 & 1.6 & 13.9 \\
\hline T3- UC 2.5 t ha $^{-1}$ & 1.5 & 0.26 & 1.40 & 19.7 & 3.4 & 18.3 & 1.50 & 0.26 & 2.08 & 16.3 & 2.8 & 22.1 & 1.43 & 0.24 & 1.83 & 12.3 & 2.1 & 15.7 \\
\hline T4- UC $5 \mathrm{t} \mathrm{ha}^{-1}$ & 1.7 & 0.36 & 1.53 & 26.4 & 5.6 & 23.7 & 1.70 & 0.27 & 2.25 & 22.3 & 3.6 & 30.4 & 1.49 & 0.25 & 2.05 & 15.6 & 2.6 & 21.5 \\
\hline T5- SS $2.5 \mathrm{t} \mathrm{ha}^{-1}$ & 1.6 & 0.42 & 1.56 & 25.6 & 6.7 & 24.9 & 1.70 & 0.27 & 2.29 & 23.7 & 3.8 & 32.3 & 1.50 & 0.25 & 2.10 & 16.2 & 2.7 & 22.7 \\
\hline T6- SS $5 \mathrm{t} \mathrm{ha}^{-1}$ & 1.8 & 0.53 & 1.60 & 34.5 & 10.1 & 30.7 & 1.70 & 0.28 & 2.37 & 26.2 & 4.3 & 36.3 & 1.60 & 0.26 & 2.22 & 19.4 & 3.1 & 26.9 \\
\hline T7- PM $2.5 \mathrm{t} \mathrm{ha}^{-1}$ & 1.7 & 0.50 & 1.58 & 27.5 & 8.1 & 25.6 & 1.70 & 0.28 & 2.37 & 25.3 & 4.2 & 35.2 & 1.51 & 0.26 & 2.15 & 17.5 & 3 & 24.9 \\
\hline T8- PM $5 \mathrm{t} \mathrm{ha}^{-1}$ & 1.9 & 0.56 & 1.64 & 38.0 & 11.2 & 32.8 & 1.80 & 0.29 & 2.48 & 29.9 & 4.9 & 41.7 & 1.64 & 0.27 & 2.28 & 21.2 & 3.5 & 29.4 \\
\hline T9- UC $2.5 \mathrm{t} \mathrm{ha}^{-1}+75 \%$ RDF & 1.8 & 0.35 & 1.51 & 35.8 & 6.9 & 30.0 & 1.80 & 0.29 & 2.36 & 26.7 & 4.4 & 35.4 & 1.51 & 0.25 & 2.00 & 15.4 & 2.6 & 20.4 \\
\hline T10- UC $5 \mathrm{t} \mathrm{ha}^{-1}+75 \%$ RDF & 2.1 & 0.42 & 1.58 & 51.0 & 10.2 & 38.4 & 1.80 & 0.31 & 2.45 & 30.1 & 5.1 & 40.6 & 1.54 & 0.26 & 2.18 & 16.9 & 2.9 & 24.0 \\
\hline T11- SS $2.5 \mathrm{t} \mathrm{ha}^{-1}+75 \%$ RDF & 2 & 0.45 & 1.60 & 50.6 & 11.3 & 40.4 & 1.80 & 0.31 & 2.49 & 32.8 & 5.7 & 45.6 & 1.60 & 0.26 & 2.20 & 20.5 & 3.3 & 28.2 \\
\hline T12-SS $5 \mathrm{t} \mathrm{ha}^{-1}+75 \%$ RDF & 2.2 & 0.56 & 1.66 & 59.8 & 15.2 & 45.2 & 1.90 & 0.33 & 2.85 & 37.4 & 6.5 & 56.1 & 1.66 & 0.27 & 2.38 & 23.4 & 3.8 & 33.6 \\
\hline T13- PM $2.5 \mathrm{t} \mathrm{ha}^{-1}+75 \%$ RDF & 2.1 & 0.53 & 1.61 & 55.0 & 13.8 & 42.2 & 1.90 & 0.32 & 2.57 & 33.1 & 5.6 & 45.0 & 1.60 & 0.26 & 2.33 & 21.0 & 3.4 & 30.6 \\
\hline T14- PM $5 \mathrm{t} \mathrm{ha}^{-1}+75 \%$ RDF & 2.3 & 0.58 & 1.68 & 67.4 & 16.5 & 49.2 & 2.00 & 0.34 & 3.00 & 42.2 & 7.1 & 63.0 & 1.71 & 0.28 & 2.63 & 28.0 & 4.6 & 43.1 \\
\hline Mean & 1.9 & 0.44 & 1.60 & 38.9 & 9.3 & 32.0 & 1.70 & 0.29 & 2.40 & 27.2 & 4.6 & 37.8 & 1.53 & 0.25 & 2.12 & 17.8 & 2.9 & 24.9 \\
\hline S.E m $( \pm)$ & 0.04 & 0.05 & 0.08 & 2.3 & 0.3 & 0.4 & 0.04 & 0.01 & 0.04 & 0.42 & 0.0 & 0.4 & 0.01 & 0.01 & 0.07 & 0.6 & 0.1 & 0.4 \\
\hline $\mathrm{CD}(0.05)$ & 0.11 & 0.16 & 0.25 & 6.6 & 0.9 & 1.2 & 0.13 & 0.04 & 0.11 & 1.21 & 0.1 & 1.1 & 0.04 & 0.02 & 0.20 & 1.7 & 0.2 & 1.1 \\
\hline
\end{tabular}

Note: RDF: recommended dose of fertilizer UC: urban compost SS: sewage sludge PM: poultry manure 
Table.6 Total economics of brinjal -cauliflower cropping sequence during 2013-2014

\begin{tabular}{|c|c|c|c|}
\hline Treatments & $\begin{array}{c}\text { Total cost of } \\
\text { cultivation (Rs) }\end{array}$ & $\begin{array}{c}\text { Total net } \\
\text { returns (Rs) }\end{array}$ & B:C ratio \\
\hline $\mathrm{T}_{1}$ & 124855 & 75145 & 0.60 \\
\hline $\mathrm{T}_{2}$ & 129667 & 241133 & 1.85 \\
\hline $\mathrm{T}_{3}$ & 125205 & 153675 & 1.22 \\
\hline $\mathrm{T}_{4}$ & 125555 & 178445 & 1.42 \\
\hline $\mathrm{T}_{5}$ & 125280 & 188720 & 1.50 \\
\hline $\mathrm{T}_{6}$ & 125705 & 224695 & 1.78 \\
\hline $\mathrm{T}_{7}$ & 127855 & 201945 & 1.57 \\
\hline $\mathrm{T}_{8}$ & 130855 & 236145 & 1.80 \\
\hline $\mathrm{T}_{9}$ & 128981 & 229219 & 1.77 \\
\hline $\mathrm{T}_{10}$ & 129331 & 251069 & 1.94 \\
\hline $\mathrm{T}_{11}$ & 129055 & 260544 & 2.01 \\
\hline $\mathrm{T}_{12}$ & 129481 & 295119 & 2.27 \\
\hline $\mathrm{T}_{13}$ & 131631 & 267569 & 2.03 \\
\hline $\mathrm{T}_{14}$ & 134631 & 301369 & 2.23 \\
\hline
\end{tabular}

In brinjal, highest $\mathrm{N}$ concentration $(2.3 \%)$ and uptake $\left(67.4 \mathrm{~kg} \mathrm{ha}^{-1}\right)$ and in cauliflower cumulative and residual, the highest $\mathrm{N}$ concentration $(2.00 \%)$ and $\mathrm{N}$ uptake $(42.2 \mathrm{~kg}$ $\mathrm{ha}^{-1}$ ) was obtained with PM $5 \mathrm{t} \mathrm{ha}^{-1}+75 \% \mathrm{RD}$ (Table 5). Combined application of organic and inorganic sources of nutrients improved nutrient uptake owing to the adequate and continuous supply of nutrients. The results are in line with those of Choudary et al., (2013); Wani et al., (2010) and Narayan et al., (2004). Integrated application of manures and fertilizers showed significant effect on $\mathrm{P}$ concentration and uptake, In both the seasons, poultry manure $5.0 \mathrm{t} \mathrm{ha}^{-1}+75 \% \mathrm{RDF}$ recorded highest $\mathrm{P}$ content and $\mathrm{P}$ uptake (Table 5).

Poultry manure treated plots recorded the higher $\mathrm{P}$ uptake than other sources. The addition of poultry manure might have helped in elongation of root system and ramification of roots in search of nutrients led to increased uptake of nutrients. Application of organic manures along with fertilizers showed significant effect on $\mathrm{K}$ concentration and uptake, among the treatments, the highest $\mathrm{K}$ concentration and uptake was obtained in plant with poultry manure $5.0 \mathrm{t} \mathrm{ha}^{-1}+75 \%$ RDF (Table 5). Poultry manure treated plots recorded the higher uptake of $\mathrm{K}$ than other treatments; this might be due to increased availability of nutrients from mineralized organic manure, which increased the concentration of $\mathrm{K}$ in soil solution in a readily available form for absorption.

\section{Economics of brinjal- cauliflower cropping sequence}

Highest values for net income noted for treatment involving combined use of poultry manure 5t ha ${ }^{-1}+75 \%$ RDF (Rs.301369 / ha), whereas the highest benefit: cost ratio (2.27) were recorded with sewage sludge $5 \mathrm{t} \mathrm{ha}^{-1}+$ 75\% RDF (Table 6). On an overall view, 
integrated treatments performed better than alone manure applications.

It can be concluded that in both the seasons, integrated application of manures and fertilizers recorded superior yields and uptake of nutrients. Among the treatments higher yields, nutrient concentration and uptake were recorded with application of poultry manure 5 $\mathrm{t} \mathrm{ha}^{-1}$ along with 75 percent RDF. Since the experimental soil is poor in fertility, combined use of organic and inorganic sources helped the crops through improved nutrition and also by maintaining soil productivity for longer period of time.

\section{References}

Choudary H.R., O.P. Sharma, R.K. Singh, Kanchan Singh, Rakesh Kumar and Lalji Yadav. 2013. Influence of Organic Manures and Chemical Fertilizer on Nutrient uptake, Yield and Profitability of Mungbean. Madras Agri. J., 100(13): 747-750.

Kumar Mukesh, Yaduvanshi, N.P.S. and Singh, Y.V. 2012. Effects of integrated nutrient management on rice yield, nutrient uptake and soil fertility status in reclaimed sodic soils. J. Indian Soc. Soil Sci., 60: 132-137.

Laxminarayana, Susan John, Ravindran, C.S. and Naskar, S.K. 2011. Effect of lime, inorganic and organic sources on soil fertility, yield, quality and nutrient uptake of sweet potato in Alfisols. Commun. Soil Sci. Plant Analysis, 42(20): 251-252.

Narayan, S., Nazir, A., Narayan, R. and Singh, A.K. 2004. Effect of organic manures vs. inorganic fertilizers on fruit yield of tomato. In: Book of abstracts. National seminar on diversification of Agriculture through Horticultural crops, 21-24 Feb., IARI Station, Karnal.

Panse, V.G and Sukhatme, P.V. 1978. Statistical methods for Agricultural workers. Indian Council of Agricultural Research, New Delhi.

Rakhonde, M.G., D.M. Naik and Garud, H.S. 2015. Yield and quality of brinjal influenced by organic and inorganic fertilizers. Int. J. Trop. Agri., 33(3): 2303-2305.

Singh Rana Veer, Prakash Chandra, Tripath, S.K., Sharma Babloo, Singh, R.P. 2015. Effect of integrated nutrient management on productivity, nutrient uptake and economics of green gram (Vigna radiata L.) in custard apple under rainfed condition. J. Prog. Agri., 6(1): 23-26.

Wani, A.J., T. Mubarak and J.A. Bhat. 2010. Effect of integrated nutrient management on curd yield, quality and nutrient uptake of cauliflower under temperate Kashmir conditions. Crop Res., 40(1,2\&3): 109-112.

\section{How to cite this article:}

Saikumar, R. and Jeevan Rao, K. 2017. Effect of Urban Compost, Sewage Sludge and Poultry Manure with Chemical Fertilizers on Yield, Nutrient Content and Uptake in BrinjalCauliflower Cropping System. Int.J.Curr.Microbiol.App.Sci. 6(6): 1313-1321. doi: https://doi.org/10.20546/ijcmas.2017.606.154 